\title{
Stable Polyelectrolyte Multilayer-Based Hollow Fiber Nanofiltration Membranes for Produced Water Treatment
}

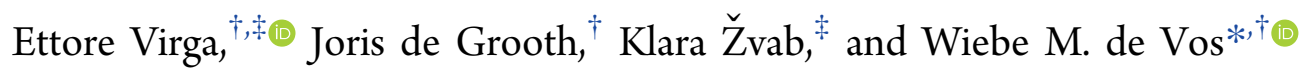 \\ ${ }^{\dagger}$ Membrane Science and Technology, University of Twente, Drienerlolaan 5, 7522 NB Enschede, The Netherlands \\ ${ }^{\ddagger}$ Wetsus, European Centre of Excellence for Sustainable Water Technology, Oostergoweg 9, 8911 MA Leeuwarden, The \\ Netherlands
}

Supporting Information

\begin{abstract}
Produced water (PW) constitutes a massive environmental issue due to its huge global production as well as its complexity and toxicity. Membrane technology could, however, convert this complex waste stream into an important source of water for reuse, but new and more efficient membranes are required. In particular, in the last few years, polyelectrolyte multilayers (PEMs) established themselves as a very powerful method to prepare hollow fiber-based nanofiltration (NF) membranes, and this membrane type and geometry would be ideal for PW treatment. Unfortunately, the presence of surfactants in PW can affect the stability of polyelectrolyte multilayers. In this work, we investigate the stability of polyelectrolyte multilayers toward different types of surfactant, initially on model surfaces. We find that chemically stable multilayers such as poly(diallyldimethylammonium chloride) (PDADMAC)/poly(sodium 4-styrenesulfonate) (PSS), based only on electrostatic interactions, are substantially desorbed by charged surfactants. For poly(allylamine hydrochloride) (PAH)/PSS multilayers, however, we demonstrate that chemical cross-linking by glutaraldehyde leads to surfactant stable layers. These stable PEM coatings can also be applied on hollow fiber support membranes to create hollow fiber NF membranes dedicated for PW treatment. Increased cross-linking time leads to more stable and more selective separation performance. These newly developed membranes were subsequently studied for the treatment of synthetic PW, consisting of freshly prepared oil-in-water emulsions stabilized by hexade-cyltrimethylammonium bromide (CTAB) and sodium dodecyl sulfate (SDS) in the presence of a mixture of ions. For both types of produced water, the membranes show excellent oil removal $(\sim 100 \%)$ and organics removal (TOC reduced up to $\sim 97 \%)$ as well as good divalent ion retentions $\left(\sim 75 \%\right.$ for $\mathrm{Ca}^{2+}$ and up to $\sim 80 \%$ for $\left.\mathrm{SO}_{4}^{2-}\right)$. Moreover, we observe a high flux recovery for both emulsions ( $100 \%$ for CTAB and $80 \%$ for SDS) and especially for the CTAB emulsion a very low degree of fouling. These stable PEM-based hollow fiber membranes thus allow simultaneous deoiling and removal of small organic molecules, particles, and divalent ions in a single step process while also demonstrating excellent membrane cleanability.

KEYWORDS: nanofiltration, produced water treatment, polyelectrolyte multilayer, surfactants for oil recovery, membrane fouling
\end{abstract}

\section{INTRODUCTION}

Water scarcity is a major global problem and is expected to only worsen because of world population growth and global warming. ${ }^{1,2}$ One of the essential steps to be taken is to find effective and efficient methods to allow wastewater reuse. ${ }^{3}$ In oil producing areas such as the Middle East, Africa, and the United States, a lot of wastewater is produced during oil extraction, counting only in the United States 3.3 million $\mathrm{m}^{3}$ in 2007. ${ }^{4}$ This so-called produced water (PW) stems from water naturally contained in the well but also from additional water pumped into the reservoir to facilitate the extraction of oil. ${ }^{5}$ Because this process generally results in three barrels of wastewater per barrel of oil extracted, ${ }^{6}$ produced water treatment could be an important source of water for various beneficial reuses, for example irrigation.
Unfortunately, PW is a very challenging stream to treat: its composition varies widely from one location to another and typically includes dispersed oil, organic and inorganic contaminants, treatment chemicals from the oil production process (e.g., corrosion and scale inhibitors, surfactants, biocides, etc.), produced solids, bacteria, metals, and naturally occurring radioactive materials. ${ }^{4,7}$ Currently, PW is treated with a wide variety of physical, chemical, and biological techniques in which hydrocyclones, evaporation, and different types of filters are employed. ${ }^{8,9}$ In such large treatment processes, membrane technology is especially interesting as it is one of the few techniques that can successfully remove the

Received: June 4, 2019

Accepted: July 11, 2019

Published: July 11, 2019 
smallest $(<10 \mu \mathrm{m})$ and most stable oil droplets. ${ }^{10}$ For this purpose, microfiltration (MF) and especially ultrafiltration (UF) have been shown to be very suitable techniques with UF also being well-suited to remove small suspended particles and even part of the dissolved hydrocarbons. ${ }^{11}$ But, membrane technology can do much more. Reverse osmosis (RO) can be used to desalinate produced water and remove final traces of dispersed and dissolved oil. ${ }^{12}$ Unfortunately, the low permeability and high pressure associated with RO translate into a larger footprint and amount of energy consumed. ${ }^{13}$ Here, nanofiltration (NF) can be seen as a very interesting alternative. NF membranes can be used at acceptable permeability for deoiling while simultaneously removing multivalent ions, dissolved organics, and part of the monovalent salts. ${ }^{14}$

Membrane technology also has clear downsides, and in PW treatment, membrane fouling can be considered the most serious problem. In the complex mixture that is produced water, many of its components can foul the membrane, leading to very substantial decreases in the flux of treated water. Fouling is especially a problem for RO and NF membranes that, due to their typical spiral wound configuration, can only deal with relatively clean feed streams. As a result, a very substantial pretreatment such as MF or UF is needed before RO and NF can be applied.

The need for a pretreatment step excludes the possibility of the proposed one step NF membrane operation to simultaneously deoil and control the salinity. If, instead, hollow fiber (HF) nanofiltration membranes could be employed, those problems can be overcome because of their ability to withstand much higher fouling loads. This is partly because HF membranes can be cleaned much better by physical cleaning than their spiral wound counterparts, ${ }^{15}$ e.g. by allowing backwashing at higher pressures. ${ }^{16}$ An equally important argument is that HF-based membrane modules do not require a spacer, while for spiral wound modules, spacer fouling is a much bigger problem than membrane fouling. ${ }^{15}$

In the past decade, polyelectrolyte multilayers (PEMs) have really established themselves as a very powerful method to prepare hollow fiber-based NF membranes. Moreover, PEMs have significant advantages that might make them especially well-suited to design membranes for produced water treatment. First, NF membranes based on PEMs are highly versatile, as the separation layer properties such as inner molecular structure and charge can be controlled by the choice of the polyelectrolytes, the number of layers, the $\mathrm{pH}$, and the ionic strength of the coating solution. ${ }^{17,18}$ Thus, it becomes possible to tune the separation performance of these membranes for a specific application or feed stream. A second advantage is the thin separation layer allowing membrane permeabilities to be relatively high compared to commercial NF membranes with comparable separation performance. ${ }^{19,20}$ Finally, polyelectrolyte multilayers coated on top of UF supports have been shown to be stable both against physical (e.g., backflushing) and chemical cleaning (hypochlorite $(\mathrm{NaOCl})) .{ }^{16}$ In contrast, conventional NF membranes based on polyamide thin film composites showed a significant drop in performance after prolonged exposure to hypochlorite. ${ }^{21}$ It is highly likely that chemical cleaning is a necessity for the treatment of produced water by any membrane type.

However, polyelectrolyte multilayer-based membranes also have weaknesses. The main challenge for PEM-based membranes, especially regarding produced water treatment, is that the multilayer stability can be compromised by the presence of charged organic molecules in the feed stream. In particular, work carried out recently showed that surfactants (naturally present in PW but also added in extra quantities to inhibit pipe corrosion and increase oil recovery) can affect the stability of common PEMs. ${ }^{22}$ As polyelectrolyte multilayers are prepared through electrostatic assembly, the major forces involved in keeping those layers together are electrostatic ones. Exposure to wastewater containing surfactant molecules, especially at high salinity, can allow the charged surfactant molecules to complex with the polyelectrolyte layers, thereby compromising the stability of the multilayer.

In recent years, some studies have focused on covalent layerby-layer films to achieve improved polyelectrolyte multilayer stability. Though exploitation of the chemistry of the chosen polyelectrolytes, it becomes possible to make stable multilayers by introducing chemical bonding via chemical cross-linking. ${ }^{23}$ Cross-linking of multilayers has been studied before, especially to increase membrane selectivity ${ }^{24}$ and sometimes also to increase chemical stability (hypochlorite) ${ }^{25}$ as well as stability in extremely saline conditions. Unfortunately, all of this previous work has focused on flat sheet membranes rather than hollow fiber-based membranes, where techniques such as UV cross-linking ${ }^{23,24}$ and multilayer spray-assisted assembly ${ }^{25}$ cannot be applied.

In this work, we report on the development of PEM-based hollow fiber NF membranes specifically for the challenging treatment of produced water. First, the focus is on the active layer stability, and we studied on model surfaces how two types of multilayer [poly(diallyldimethylammonium chloride) (PDADMAC)/poly(sodium 4-styrenesulfonate) (PSS) and poly(allylamine hydrochloride) (PAH)/PSS] are affected by exposure to surfactants with a variety of properties (cationic, anionic, zwitterionic, and nonionic). We then studied how different cross-linking approaches, concentration, and time can lead to PAH/PSS-based PEM coatings stable in surfactant solutions. The effect of cross-linking time was subsequently studied for PEM-based hollow fiber NF membranes, where the retention of a variety of salts was studied before and after surfactant exposure. Finally, through filtration experiments carried out with synthetic produced water, we demonstrated that hollow fiber-based NF membranes are able to simultaneously remove oil droplets, surfactants, and divalent ions from the waste stream in a single separation step.

\section{EXPERIMENTAL PROCEDURES}

2.1. Materials. $\mathrm{PAH}\left(M_{\mathrm{w}}=50 \mathrm{kDa}\right)$, PSS $\left(M_{\mathrm{w}}=70 \mathrm{kDa}\right)$, an aqueous solution of $20 \mathrm{wt} \% \operatorname{PDADMAC}\left(M_{\mathrm{w}}=400-500 \mathrm{kDa}\right)$, an aqueous solution of 25 wt \% glutaraldehyde (Grade II), Nmethylpyrrolidon, and glycerin were purchased from Sigma-Aldrich (The Netherlands). Poly(ether sulfone) (PES) (Ultrason 6020) and SPES (sulfonated polysulfone) were both obtained from BASF (Germany). Sodium dodecyl sulfate (SDS, Sigma-Aldrich), hexadecyltrimethylammonium bromide (CTAB, Sigma-Aldrich), $\mathrm{N}$-dodecyl$N, N$-dimethyl-3-ammonio-1-propanesulfonate (DDAPS, Sigma-Aldrich), and Triton X-100 (TX, Sigma-Aldrich) were the four types of surfactant used (Figure 1). N-Hexadecane (Merck Schuchardt) was used as the oil, and Coumarin 6/Neeliglow Yellow 196 (Neelikon) was used as the fluorescent dye to allow accurate measurements of the oil concentration. All other chemicals were purchased from VWR (The Netherlands).

2.2. Polyelectrolyte Multilayer Coating on $\mathrm{SiO}_{2}$ Model Surfaces. Silicon wafers were first cleaned with piranha solution made of a 3:1 mixture of concentrated sulfuric acid $\left(\mathrm{H}_{2} \mathrm{SO}_{4}\right)$ with hydrogen peroxide $\left(\mathrm{H}_{2} \mathrm{O}_{2}\right)$ to remove any organic residues. Then, the 


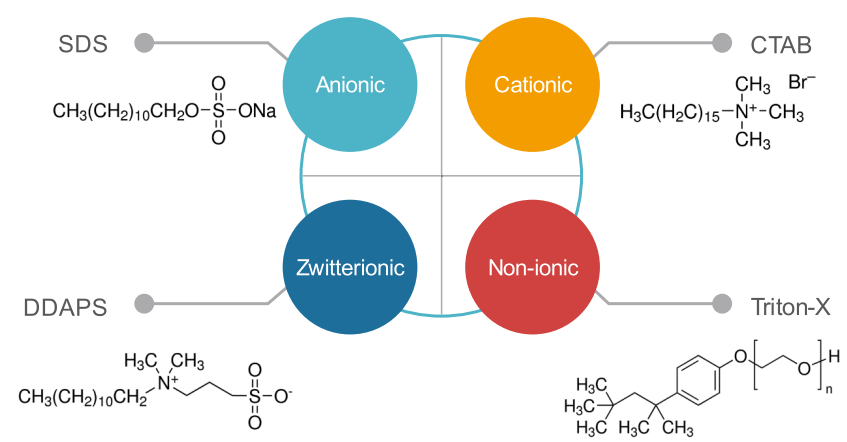

Figure 1. Surfactants used in this study: SDS, CTAB, DDAPS, and TX.

wafers were coated via a simple dipcoating procedure. In particular, $\mathrm{PAH} / \mathrm{PSS}$ and PDADMAC/PSS multilayers were coated on top of silicon wafers with 84 and $121 \mathrm{~nm} \mathrm{SiO}{ }_{2}$ top layers $\left(\delta_{\mathrm{SiO}_{2}}\right)$ respectively. These $\mathrm{SiO}_{2}$ layers are required as an optical spacer in reflectometry (see Section 2.3). For a single bilayer coating, the negatively charged silica wafer was completely immersed in a $0.1 \mathrm{~g} \cdot \mathrm{l}^{-1}$ polycation solution with $50 \mathrm{mM} \mathrm{NaCl}$. After $15 \mathrm{~min}$, the surfaces were rinsed in a $50 \mathrm{mM}$ $\mathrm{NaCl}$ solution for $15 \mathrm{~min}$. To finally complete the bilayer coating, the surfaces were subsequently immersed in a $0.1 \mathrm{~g} \cdot \mathrm{l}^{-1}$ polyanion solution (pH 5.4) with $50 \mathrm{mM} \mathrm{NaCl}$ for $15 \mathrm{~min}$ and then rinsed in a $50 \mathrm{mM}$ $\mathrm{NaCl}$ solution, again for $15 \mathrm{~min}$. The same procedure was repeated four more times to realize 4.5 and 5.0 bilayers on top of the wafers. Every coating step was executed at room temperature. For crosslinked PAH/PSS multilayers, a cross-linking step was added to the procedure written above. In particular, after every PAH coating step, starting from the second bilayer, the wafers were rinsed in $50 \mathrm{mM}$ $\mathrm{NaCl}$ and then completely immersed in aqueous solutions with various GA concentrations $(2.5,5,7.5,10$, and $15 \mathrm{mM})$ for $15 \mathrm{~min}$ at room temperature, keeping the concentration of $\mathrm{NaCl}$ to $50 \mathrm{mM}$. After this step, the wafers were carefully rinsed in $50 \mathrm{mM} \mathrm{NaCl}$ for 15 min, and then the PSS layer was applied to complete the bilayer, following the procedure described before. Surfaces coated with 4.5 bilayers of PAH/PSS at different cross-linking times were realized first via dip coating, and then they were dipped in a $7.5 \mathrm{mM}$ GA solution for different times: $1,5,15,60,180$, and $300 \mathrm{~min}$. In this last approach, membranes were thus cross-linked in a single step rather than after every PAH step, as was done in the first approach.

2.3. Reflectometry. The multilayer desorption from silicon wafers due to the interaction with surfactants was monitored by means of reflectometry. ${ }^{26}$ Cationic (CTAB, $346 \mathrm{mg} \cdot \mathrm{l}^{-1}$ ), anionic (SDS, 2391 $\mathrm{mg} \cdot \mathrm{l}^{-1}$ ), zwitterionic (DDAPS, $1006 \mathrm{mg} \cdot \mathrm{l}^{-1}$ ), and nonionic (TX, 144 $\left.\mathrm{mg} \cdot \mathrm{l}^{-1}\right)$ surfactant solutions, approximately at their critical micelle concentration (CMC) and with $5 \mathrm{mM} \mathrm{NaCl}$ as background electrolyte, were prepared. These solutions were flushed under a stagnation point flow to a silica surface and precoated with a PEM (see procedure above) several times with a $5 \mathrm{mM} \mathrm{NaCl}$ rinsing step in between until a steady state desorption was reached. The use of a stagnation point flow cell allows for very well-controlled hydrodynamics during the multilayer desorption. Polarized monochromatic light $(\mathrm{He}-\mathrm{Ne}$ laser, $632.8 \mathrm{~nm})$ hits the wafer around the Brewster angle and is reflected toward the detector. The reflected light is split into its $\mathrm{p}$ - and s- polarized components. The ratio between these two components is defined as $S(-)$, and the change in this ratio $(\Delta S)$ is directly proportional to the amount of mass desorbed from the wafer:

$$
\Gamma=\frac{\Delta S}{S_{0}} Q
$$

where $\Gamma$ is the amount of mass adsorbed or desorbed from the wafer $\left(\mathrm{mg} \cdot \mathrm{m}^{-2}\right), S_{0}$ is the starting output signal of the bare silicon wafer $(-)$, and $Q$ is the sensitivity factor for the system $\left(\mathrm{mg} \cdot \mathrm{m}^{-2}\right)$. To calculate the sensitivity factor, we used an optical model based on the following values: $\theta=71^{\circ}, n_{\text {silica }}=1.46, \tilde{n}_{\text {silicon }}=(3.85,0.02), n_{\mathrm{H}_{2} \mathrm{O}}=$
1.33, $\mathrm{d} n / \mathrm{d} c_{\text {PDADMAC }}=0.18 \mathrm{~mL} \cdot \mathrm{g}^{-1}, \mathrm{~d} n / \mathrm{d} c_{\text {PAH }}=0.16 \mathrm{~mL} \cdot \mathrm{g}^{-1}, \mathrm{~d} n / \mathrm{d} c_{\text {PSS }}$ $=0.18 \mathrm{~mL} \cdot \mathrm{g}^{-1}, \delta_{\mathrm{SiO}_{2}}=84 \mathrm{~nm}$ for PAH/PSS and $121 \mathrm{~nm}$ for PDADMAC/PSS. The sensitivity factor, $Q$ obtained to calculate the actual mass desorption for all experiments is $30 \mathrm{mg} \cdot \mathrm{m}^{-2}$ for PAH/PSS and $40 \mathrm{mg} \cdot \mathrm{m}^{-2}$ for PDADMAC/PSS. Furthermore, we defined the stability of the multilayer as given by

$$
\mathrm{PEM}_{\text {stability }}=\left(1-\frac{\Gamma_{\mathrm{des}}}{m_{\mathrm{tot}}}\right)
$$

where $\Gamma_{\text {des }}$ is the quantity in $\mathrm{mg} \cdot \mathrm{m}^{-2}$ of PEM desorbed from the wafers and monitored via reflectometry, while $m_{\text {tot }}$ is the amount $(\mathrm{mg}$. $\mathrm{m}^{-2}$ ) of PEM contained in the multilayer prior desorption, measured via reflectometry. ${ }^{17}$ All experiments were performed at least twice.

2.4. Membrane Fabrication, Modification, and Characterization. Hollow fiber membranes were produced by a spinning process based on nonsolvent induced phase separation. For this, a dope was prepared by mixing $140 \mathrm{~g}$ of PES, $70 \mathrm{~g}$ of SPES, and $120 \mathrm{~g}$ of glycerin in $650 \mathrm{~g}$ of NMP. The clear dope was then filtered through a $5 \mu \mathrm{m}$ metallic mesh and deaerated overnight. The hollow fiber membranes were spun by extruding the dope solution through a hollow fiber spinneret at $70{ }^{\circ} \mathrm{C}$ (see Figure 2). A mixture of $10 \%$

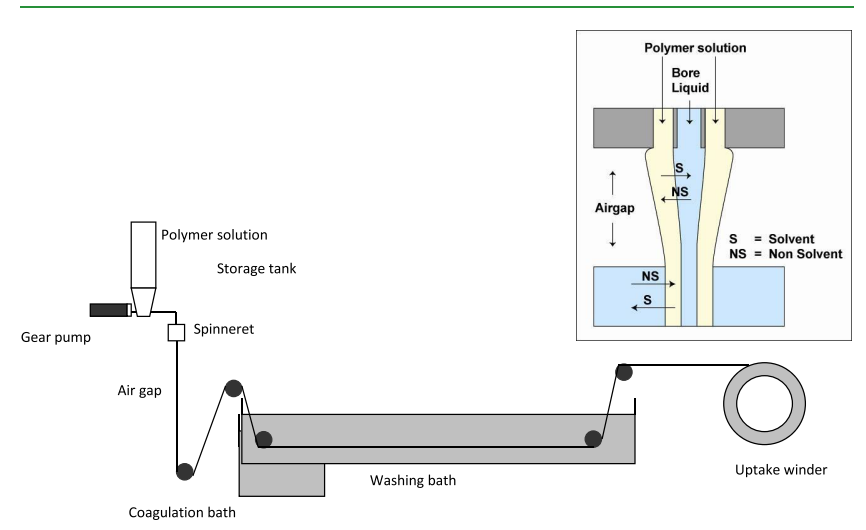

Figure 2. Diagram of the spinning setup. The inset shows the nonsolvent induced phase separation process in detail.

glycerin in water was used as the bore liquid. After an airgap of $0.3 \mathrm{~m}$, the partly already solidified membrane was immersed in the coagulation bath containing the water at $60{ }^{\circ} \mathrm{C}$. Here, the phase inversion is completed. After the coagulation bath, the fiber was fed through a washing bath with water at room temperature. Finally, the fiber was collected on an uptake winder.

These hollow fiber membranes are designed for inside-out filtration, having the smallest pores at the inside of the fiber. Scanning electron microscope (SEM) pictures of the membrane are reported in the Supporting Information (Figure S1). These HF membranes have a water permeability of $150 \mathrm{~L} \cdot \mathrm{m}^{-2} \cdot \mathrm{h}^{-1} \cdot \mathrm{bar}^{-1}$, an inner diameter of 0.7 $\mathrm{mm}$, and a molecular weight cutoff (MWCO) of $7.5 \mathrm{kDa}$. The MWCO was determined by using a polyethylene glycol (PEG) mixture with various molecular weights $(6,10,20$, and $35 \mathrm{kDa})$ and analyzing the molecular weight above which a $90 \%$ retention was obtained. For this, permeate and retentate samples were analyzed using size exclusion chromatography (SEC). After fabrication, the membranes were stored in demi-water.

Polyelectrolyte multilayers were coated on top of the prepared UF membranes. As we immerse the fibers completely in the coating solution, the deposition of polyelectrolyte is not limited to the inner surface of the membrane only and the whole porous structure can be coated by the polyelectrolytes. However, only at the inner surface, where the pores are smallest, does the PEM layer form a dense separation layer on top of the membranes. ${ }^{16-18}$ Before proceeding with the coating, we first immersed the fibers for $15 \mathrm{~min}$ in a $50 \mathrm{mM}$ $\mathrm{NaCl}$ solution. The membranes were then coated via a simple dipcoating procedure. For the bilayer coatings, the fibers were 

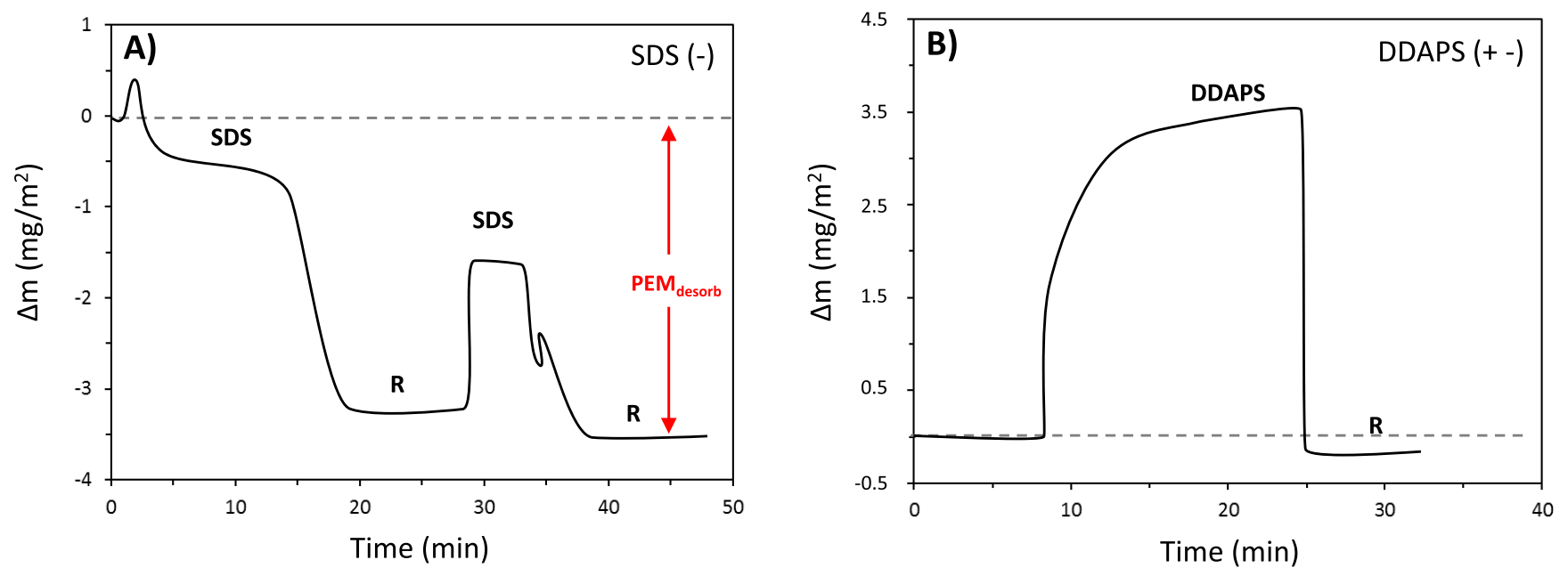

Figure 3. Variation in PEM mass $\left(\mathrm{mg} / \mathrm{m}^{2}\right)$ for a PDADMAC/PSS system after flushing with (A) SDS and (B) DDAPS at their CMC. Results were obtained via reflectometry.

completely immersed in a $0.1 \mathrm{~g} \cdot \mathrm{l}^{-1} \mathrm{PAH}$ solution with $50 \mathrm{mM} \mathrm{NaCl}$ at room temperature. After $15 \mathrm{~min}$, the fibers were rinsed in a $50 \mathrm{mM}$ $\mathrm{NaCl}$ solution for $15 \mathrm{~min}$. The fibers were subsequently dipped for 15 min in $0.1 \mathrm{~g} \cdot \mathrm{l}^{-1}$ PSS solution with $50 \mathrm{mM} \mathrm{NaCl}$ and then rinsed for another $15 \mathrm{~min}$ in a $50 \mathrm{mM} \mathrm{NaCl}$ solution. After this first bilayer, the described procedure was repeated until 4.5 and 5.0 bilayers had been coated on the membranes (taking samples for those two steps). In the case of cross-linked multilayers, fibers coated with 4.5 bilayers were immersed in a $7.5 \mathrm{mM}$ GA solution for 1,3 , and $5 \mathrm{~h}$, keeping the same ionic strength of the other steps, i.e. $50 \mathrm{mM} \mathrm{NaCl}$. After this crosslinking step, the fibers were rinsed in $50 \mathrm{mM} \mathrm{NaCl}$, and part of them were dipped in a $0.1 \mathrm{~g} \cdot \mathrm{l}^{-1}$ PSS solution with $50 \mathrm{mM} \mathrm{NaCl}$. This way, it was possible to obtain fibers with 4.5 (PAH terminated) and 5.0 (PSS terminated) bilayers at different cross-linking times. After being rinsed in demi-water, all membrane samples were put in a glycerol/ water $(15 / 85 \mathrm{wt} \%)$ mixture for $4 \mathrm{~h}$ and dried overnight under ambient conditions.

For water permeability and ion retention experiments, single PEMcoated fibers were potted in modules with a fiber length of approximately $150 \mathrm{~mm}$. Every module contains only one fiber. The water permeability $\left(\mathrm{L} \cdot \mathrm{m}^{-2} \cdot \mathrm{h}^{-1} \cdot \mathrm{bar}^{-1}\right)$ was calculated by normalizing the measured pure water flux with the transmembrane pressure (TMP). The pure water flux was measured at $20^{\circ} \mathrm{C}$ with demi-water in a cross-flow configuration at a transmembrane pressure of 3 bar. For the salt retention measurements, a cross-flow through the fibers was also applied. To reduce the effect of concentration polarization, we set the cross-flow velocity of the feed through the fibers at $1.7 \mathrm{~m}$. $\mathrm{s}^{-1}$. This corresponds to a Reynolds number of approximately 1200 . The salt concentrations of the feed and permeate were measured with a WTW Cond 3210 conductivity meter. The retention was based on the ratio between the permeate and feed concentrations. All experiments were performed at least in triplicate.

2.5. Membrane Stability. Single PEM-coated membrane fibers with 4.5 and 5 bilayers of PAH/PSS, prepared at different crosslinking times $(0 \mathrm{~h}$, i.e. not cross-linked, and 1,3 , and $5 \mathrm{~h})$ were potted in modules with a fiber length of approximately $150 \mathrm{~mm}$. Every module contains only one fiber. After each fiber was flushed three times with alternating SDS and CTAB solutions (at their CMC with 5 $\mathrm{mM} \mathrm{NaCl}$ as background electrolyte), the permeability and ion retention of the fibers were measured using the same flow and pressure conditions previously described. Each single surfactant flush lasted $5 \mathrm{~min}$. All experiments were performed at least in triplicate.

2.6. Artificial Produced Water: Preparation and Filtration. To detect the amount of oil that permeates through the membrane, $n$ hexadecane was colored with a fluorescent dye (Coumarin 6). As fluorescence decays over time, the colored oil was freshly prepared before each experiment. Around $10 \mathrm{mg}$ of the dye powder was added to $8 \mathrm{~mL}$ of $n$-hexadecane and put in an ultrasonic bath for a few minutes. Afterward, the oil was filtered with a Millipore $0.45 \mu \mathrm{m}$ filter to remove any residual solid particles. To prepare our artificial PW, we followed a procedure set out by Dickhout et al. ${ }^{27}$ To ensure all emulsions have the same characteristics, a stock emulsion was prepared under standard conditions, which was then diluted with carefully chosen concentrations to obtain the desired oil, salt, and surfactant concentrations for the filtration experiments. The surfactant concentrations were all chosen to be approximately at CMC. The stock emulsions were prepared by dissolving a surfactant $(346 \mathrm{mg} / \mathrm{L}$ CTAB or $2391 \mathrm{mg} / \mathrm{L}$ SDS) in $1 \mathrm{~L}$ of DI water in a Duran bottle by mixing with a dispersing mixer (IKA T25 digital Ultra-Turrax with S25N $18 \mathrm{G}$ element) for $4 \mathrm{~min}$ at $14000 \mathrm{rpm}$. Then, $2 \mathrm{~g}$ of colored oil was injected near the mixer head with a long syringe needle and mixed for $10 \mathrm{~min}$ at $14000 \mathrm{rpm}$. The stock emulsion was diluted to contain $1 \mathrm{~g} / \mathrm{L} n$-hexadecane and the desired surfactant and salts concentrations to make up $1 \mathrm{~L}$ of feed emulsion with $1000 \mathrm{mg} / \mathrm{L}$ $n$-hexadecane, $346 \mathrm{mg} / \mathrm{L} \mathrm{CTAB}$ or $2391 \mathrm{mg} / \mathrm{L}$ SDS, $5 \mathrm{mM} \mathrm{NaCl}, 5$ $\mathrm{mM} \mathrm{CaCl}$, and $5 \mathrm{mM} \mathrm{Na}_{2} \mathrm{SO}_{4}$. Surfactant solutions used for cleaning were prepared the same as the emulsions but without adding $n$ hexadecane. In each experiment, the concentrations of surfactant and salts in the rinsing solution were identical to the emulsion used.

For the membrane crossflow filtration experiments, single PEMcoated fibers with 4.5 bilayers were potted in modules with a fiber length of approximately $80 \mathrm{~mm}$. Every module contains only one fiber. The clean water flux of every fiber was measured before filtrating the $\mathrm{O} / \mathrm{W}$ emulsion. New modules were used for each experiment. The concentrate was recycled to the feed bottle while the permeate was instead collected. Because the permeate flow rate was small compared to the concentrate flow, this was not expected to affect the feed composition. This was checked by analyzing the feed ion concentration, TOC, and oil content at the start and end of some experiments, and indeed, negligible changes in feed composition were observed. A membrane filtration experiment consisted of filtering the $\mathrm{O} / \mathrm{W}$ emulsion for $3 \mathrm{~h}$ at a TMP of $3 \mathrm{bar}$ and a flow rate of $0.75 \mathrm{~kg} / \mathrm{h}$, which corresponds to a cross-flow velocity of $0.55 \mathrm{~cm} / \mathrm{s}$ and a Reynolds number around 380. The permeate flux was measured between 2 and $3 \mathrm{~h}$. Samples of the permeate were collected at the same time, and their ionic content and TOC were analyzed by ion chromatography (Metrohm Compact IC 761) and by a TOC analyzer (Shimadzu TOC-L), respectively. The oil retention was measured through a fluorescent dye method already used in previous work. ${ }^{28}$ Feed samples were taken at the same time as the permeate samples and analyzed with the same methods. To clean the membranes, the modules were flushed one time with a surfactant rinsing solution for $15 \mathrm{~min}$ at a $4 \mathrm{~kg} / \mathrm{h}$ flow rate without applying transmembrane pressure. After cleaning, the water flux was measured again to 
determine the flux recovery. Each experiment was performed in triplicate.

\section{RESULTS AND DISCUSSION}

The Results and Discussion Section is split into three distinct sections. In the first section, we describe application of model surfaces to study PEM stability against a variety of surfactant types and demonstrate that cross-linking can lead to stable PEM coatings even in the presence of surfactants. In the next section, we demonstrate this stability also for PEM-based hollow fiber NF membranes where ionic retentions are studied before and after cross-linking. In the final section, we show that with these membranes we can, in a single step, remove surfactants, deoil, and partially desalinate a synthetic PW stream based on a surfactant stabilized $\mathrm{O} / \mathrm{W}$ emulsion.

3.1. Multilayer Stability against Surfactants. While it is known that surfactants can destabilize PEMs, ${ }^{22}$ much less is known about how different surfactant types affect different types of PEM. Here, we studied the stability of PEMs adsorbed on model surfaces via optical flow-cell reflectometry. A wafer, precoated with a PEM, is exposed several times to a surfactant solution at the CMC until a stable plateau in the desorption is obtained. Examples of these experiments are shown in Figure 3 for exposure of a PDADMAC/PSS-based PEM to the anionic SDS (A) and the zwitterionic DDAPS (B), the outcomes of which are discussed in detail below. The desorption plateau was taken after rinsing with a rinsing solution to ensure that no adsorbed surfactant remains, as indicated in Figure 3A. All surfactant and rinsing solutions contain $5 \mathrm{mM}$ background electrolyte. Finally, the desorbed amount (in $\mathrm{mg} \cdot \mathrm{m}^{-2}$ ) can be compared to the original amount of multilayer adsorbed on the model surfaces, as discussed in Section 2.3. The stability of the PEM layer is thus defined as the percentage of the remaining mass of the original PEM coating after surfactant exposure.

We first studied the stability of a PEM based on PDADMAC/PSS, a strong polyelectrolyte multilayer couple, which is well-known in the membrane field for its high chemical stability. ${ }^{16}$ In Figure 4, we show the PEM stability after exposure to surfactants for PEMs of 4.5 bilayers (terminated with PDADMAC) and 5.0 bilayers (terminated with PSS). Here, the stability (\%) of the multilayers is reported

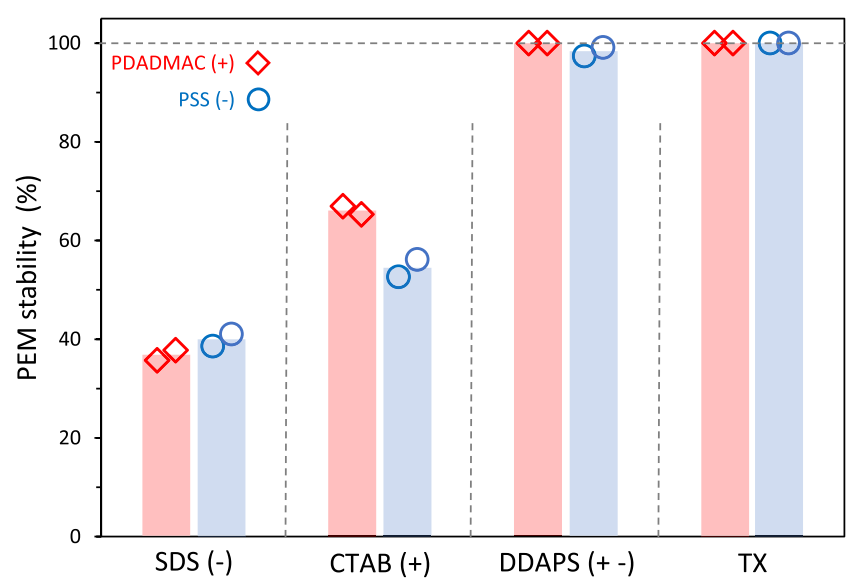

Figure 4. Stability (\%) of PDADMAC/PSS PEM with 4.5 bilayers (red) and 5 bilayers (blue) after flushing with different surfactant solutions (SDS, CTAB, DDAPS, and Triton-X) at their CMC. Results were obtained via reflectometry. Points represent single data points, while bars represent the average of these points. after exposure to four types of surfactant: anionic (SDS), cationic (CTAB), zwitterionic (DDAPS), and nonionic (TX). The effect of both a positive and a negative top layer (4.5 and 5.0 bilayers) on the stability was investigated as the charge of the top layer could affect the adsorption of the surfactant and how it interacts with the multilayer.

The results depicted in Figure 4 clearly demonstrate that charged surfactants such as the anionic SDS and the cationic CTAB compromise the stability of PDADMAC/PSS multilayers significantly. In particular, only $40 \%$ of a PDADMAC/ PSS multilayer prepared at $50 \mathrm{mM} \mathrm{NaCl}{ }^{17}$ remains after exposure to SDS, and only $60 \%$ remains after exposure to CTAB. On the other hand, surfactants carrying no net charge, such as the zwitterionic DDAPS and the nonionic TX, do not affect the stability of the multilayer.

It is well-known that charged surfactants can interact with oppositely charged polyelectrolytes to form complexes in solution. ${ }^{29}$ Polyelectrolyte multilayers are mainly based on electrostatic interactions, which can be supported by other interactions such as hydrogen bonding and hydrophobicity. ${ }^{30}$ After the addition of a charged surfactant, the small surfactant molecules can diffuse into the multilayer ${ }^{22}$ and screen the electrostatic interactions between the oppositely charged polyelectrolytes. In particular, the cationic $\mathrm{CTAB}$ could form a complex with the anionic PSS, while the anionic SDS may complex with the cationic PDADMAC. This results in competitive behavior where complexation of the surfactant with one polyelectrolyte weakens and possibly disrupts the polyelectrolyte complexation. We do find that no surfactant can completely desorb the multilayer; thus, we expect that if the desorption of one polyelectrolyte is favored, then a strong charge will build up in the multilayer. For example if negative SDS would desorb the positive PDADMAC, a significant excess of negative charge would form in the multilayer. This excess charge would then prevent more surfactant molecules from approaching the remaining PEM coating.

As PDADMAC/PSS-based multilayers are clearly not stable against desorption by charged surfactants, we moved to PSS/ PAH-based multilayers. PSS/PAH is also a very well-studied PEM system known to give dense (low hydration) layers with an excess of positive charge (PAH). Another advantage of this system is that the primary amine groups of PAH can be crosslinked by application of glutaraldehyde. ${ }^{23}$ In Figure 5, we show the stability of a PAH/PSS-based PEM without cross-linking (0 $\mathrm{mM})$ and after cross-linking with various cross-linker concentrations. Here, we cross-linked after every bilayer coating step and focus on the stability after exposure to both charged surfactants, SDS and CTAB. The experiments were again performed by using reflectometry. This time, a multilayer coated on top of a silicon wafer was first exposed to one charged surfactant, and then, after rinsing with $5 \mathrm{mM} \mathrm{NaCl}$, to the other charged surfactant, both at their CMC for at least three times. If the multilayer was terminated with a cationic PAH layer, the experiment started with exposure to the anionic SDS, while for a PEM terminated with anionic PSS, the exposure started with cationic CTAB.

From Figure 5, it is clear that without cross-linking, also the $\mathrm{PAH} / \mathrm{PSS}$ system is not stable in surfactant solution with 85$100(\%)$ of the layer being removed after consecutive exposure to SDS and CTAB. But even at very low cross-linker concentration, nearly complete stability is observed for all cross-linked layers. These GA concentrations are much lower (and thus safer) ${ }^{31}$ compared to the concentrations used by 


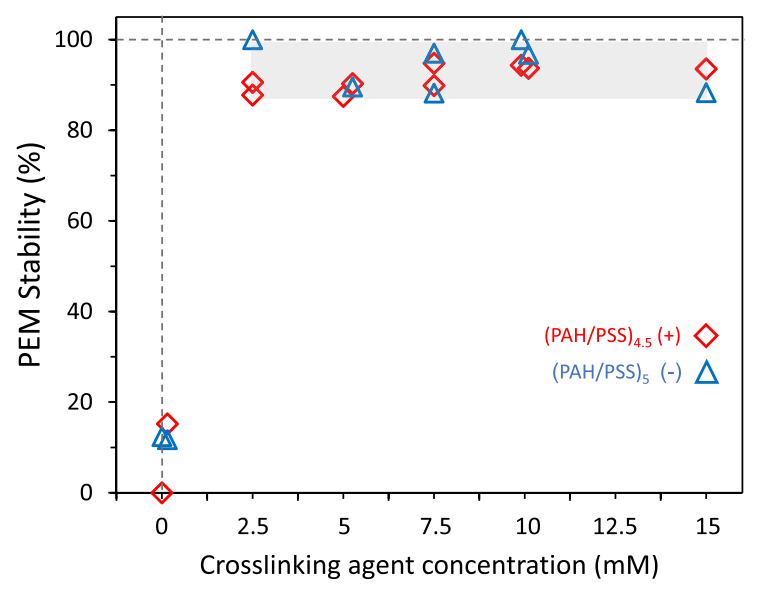

Figure 5. Stability (\%) as a function of GA concentration of a PAH/ PSS-based PEM with 4.5 bilayers (orange) or 5.0 bilayers (blue) after flushing with SDS and CTAB solutions at their CMC three times. These results were obtained by using reflectometry. Points represent single data points.

Cho et al. $(0.25-2.5 \mathrm{M}){ }^{25}$ What might come as a surprise is that typically no complete stability is observed, and that even at higher cross-linker concentrations, some material is desorbed. It is important to remember that only the PAH chains become cross-linked by glutaraldehyde, and some more loosely bound PSS chains, especially from the top layers, could still be desorbed.

The quality of cross-linking can certainly also be affected by the cross-linking time. Because cross-linking after every step (Figure 5) may not be needed, it would be very beneficial to do the cross-linking in a single final step. In Figure 6, we show the stability of a PAH/PSS multilayer, sequentially exposed to SDS and CTAB, as a function of cross-linking time. Here, cross-linking is applied in a single final step with a cross-linker concentration of $7.5 \mathrm{mM}$ and studied using the same optical reflectometry approach.

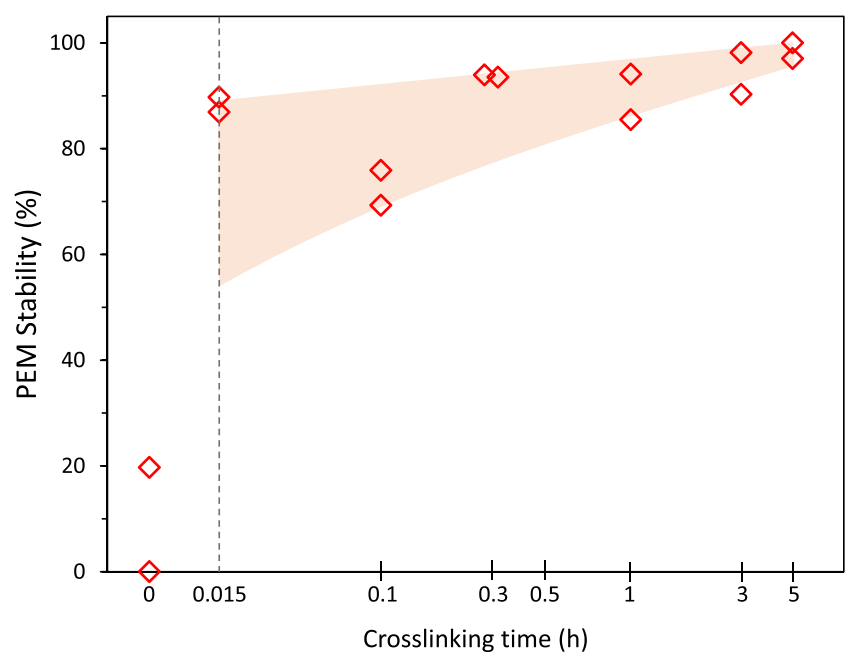

Figure 6. Stability (\%) as a function of cross-link time for a PAH/ PSS-based PEM of 4.5 bilayers after flushing with SDS and CTAB solutions at their CMC three times. These results were obtained by using reflectometry. Points represent single data points.
By looking at Figure 6, we can indeed see that, given sufficient cross-linking time, a single cross-linking step works just as well as multiple steps. This is underpinned by the later work performed on membranes (see Figure 7 and Figure 8). We also conclude that longer cross-linking times lead to more stable layers and more reproducible results.

3.2. Stable PEM-Based NF Membranes. In the previous section, we showed that PAH/PSS-based PEM coatings can be made stable by a single cross-linking step at a low cross-linker concentration. Here, we prepared hollow fiber-based NF membranes by applying PSS/PAH multilayers on top of negatively charged hollow fiber support membranes. The membranes were coated under identical conditions as the model surfaces and were subsequently cross-linked for different times using a safe gluteraldehyde concentration $(7.5 \mathrm{mM}) .{ }^{31} \mathrm{In}$ Figure 7, we show the membrane performance for PAH/PSS membranes of 4.5 (7A, PAH terminated) and 5.0 (7B, PSS terminated) bilayers in thickness. Overall, longer cross-linking times lead to a denser membrane with a lower water permeability and a higher ion retention. Similar effects were observed by Park et al. ${ }^{32}$ The increase in retention is especially strong for $\mathrm{Na}_{2} \mathrm{SO}_{4}$. We expect that here the retention is dominated by the divalent $\mathrm{SO}_{4}^{2-}$. Overall, PSS/PAH membranes tend to have an excess of positive $\mathrm{PAH}^{25}$ and a more positive charge would allow more easy permeation of this ion. But, cross-linking of glutaraldehyde with the primary amines of PAH can decrease the positive charge and even induce a negative charge in the membrane. ${ }^{25}$ This would lead to a stronger Donnan-based repulsion ${ }^{33}$ between $\mathrm{SO}_{4}^{2-}$ and the cross-linked membrane, increasing retention significantly.

The stability of these membranes is studied in Figure 8. The membranes were consecutively exposed to anionic and cationic surfactant solutions at least three times, exactly as was done for the model surfaces studied in Figures 5 and 6 . Also here, if the multilayer terminates with a positive top layer, the experiment started with SDS flushing, while if the multilayer ended with a negative top layer, the experiment started with $\mathrm{CTAB}$. Figure 8 shows the retention of $(5 \mathrm{mM}) \mathrm{CaCl}_{2}$ and $\mathrm{Na}_{2} \mathrm{SO}_{4}$ tested and compared with the retentions obtained before the surfactant flush. For the un-cross-linked PEM-based membranes $(0 \mathrm{~h})$, retentions decrease to nearly 0 , in line with the substantial desorption observed for PAH/PSS coatings on model surfaces (Figure 5). However, with an increase in cross-linking time, the reduction in retention decreases, until at $5 \mathrm{~h}$ of cross-linking, very stable performance is observed. Clearly, the stability that was observed at high cross-linking times on model surfaces (Figure 6) is also found for PEM-based membranes. For shorter cross-linking times for the $\mathrm{PAH}$ terminated membrane $(8 \mathrm{~A})$, we observed a decrease in retention of both $\mathrm{Na}_{2} \mathrm{SO}_{4}$ and $\mathrm{CaCl}_{2}$. A strange observation is that for shorter cross-linking times for the PSS terminated membrane (8B), $\mathrm{Na}_{2} \mathrm{SO}_{4}$ retention decreases and $\mathrm{CaCl}_{2}$ retention increases. Most likely, the surfactant exposure does remove some of the PSS from the PSS terminated membrane, making the layer more positively charged, leading to an increase in $\mathrm{CaCl}_{2}$ retention and a decrease in $\mathrm{Na}_{2} \mathrm{SO}_{4}$ retention.

3.3. Produced Water Treatment. A stable hollow fiber NF membrane would be highly beneficial for produced water treatment, as one could deoil and remove particles, small organic molecules (surfactants), and multivalent ions in a single step. Two artificial produced water emulsions stabilized either by SDS or CTAB with, as oil, 1000 ppm $n$-hexadecane (droplet size $\sim 5 \mu \mathrm{m}^{27}$ ) and $5 \mathrm{mM} \mathrm{NaCl}, 5 \mathrm{mM} \mathrm{CaCl}$, and 5 

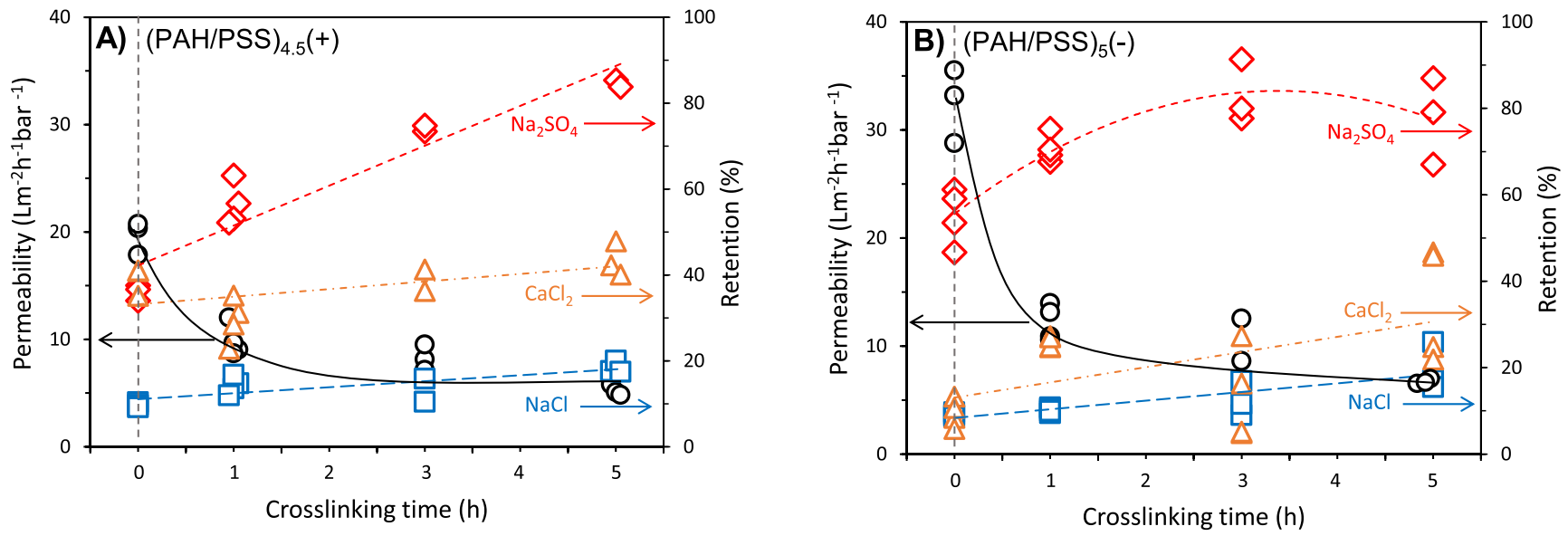

Figure 7. Water permeability $\left(\mathrm{L} \cdot \mathrm{m}^{-2} \cdot \mathrm{h}^{-1} \cdot \mathrm{bar}^{-1}\right)$ and retentions (\%) of $5 \mathrm{mM} \mathrm{CaCl}, \mathrm{NaCl}$, and $\mathrm{Na}_{2} \mathrm{SO}_{4}$ of membranes coated with (PAH/PSS) ${ }_{4}$. $\mathrm{PAH}(\mathrm{A})$ and $(\mathrm{PAH} / \mathrm{PSS})_{5}$ (B) cross-linked for $0,1,3$ and $5 \mathrm{~h}$ with GA. Points represent single data points, while lines represent the average of these points.
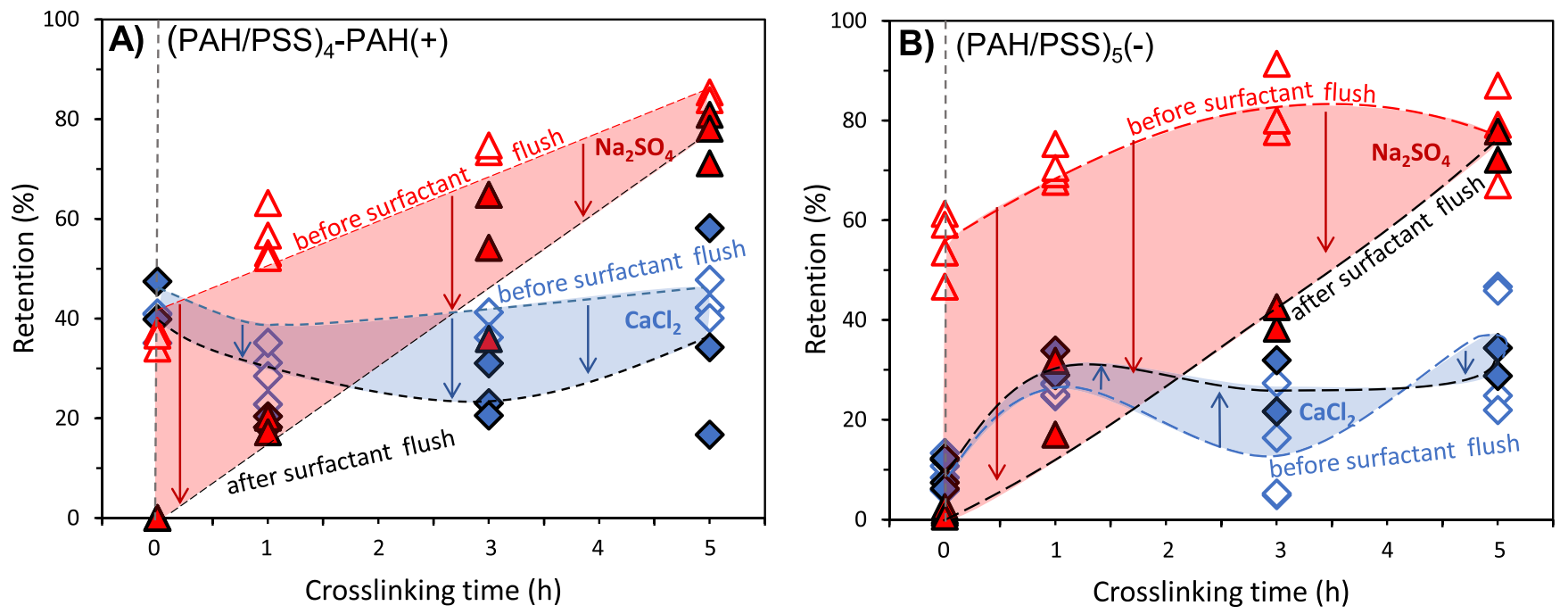

Figure 8. Retentions of $5 \mathrm{mM} \mathrm{CaCl}$ and $\mathrm{Na}_{2} \mathrm{SO}_{4}$ as a function of cross-linking time for membranes coated with (PAH/PSS) $)_{4} \mathrm{PAH}(\mathrm{A})$ and $(\mathrm{PAH} / \mathrm{PSS})_{5}(\mathrm{~B})$ before and after consecutive rinsing three times with SDS and CTAB solutions at their CMC. Data are represented by points, while lines represent the average of these points.

$\mathrm{mM} \mathrm{Na}_{2} \mathrm{SO}_{4}$, were treated in a crossflow system by using $\mathrm{HF}$ membranes based on 4.5 bilayers of PAH/PSS cross-linked for $5 \mathrm{~h}$ in $7.5 \mathrm{mM}$ glutaraldehyde. The crossflow velocity was set at $0.55 \mathrm{~cm} / \mathrm{s}$, and the TMP was kept constant at 3 bar.

In Figure 9A, we show the retentions stemming from filtrations using the cationic $\mathrm{CTAB}$ as surfactant to stabilize the $\mathrm{O} / \mathrm{W}$ emulsion. We observe an excellent oil retention (100\%) as well as a very high TOC retention (96.5\%), implying that also nearly all of the free surfactant molecules are retained. Furthermore, higher $\mathrm{Ca}^{2+}(75 \%)$ and $\mathrm{Cl}^{-}$(36\%) retentions were obtained compared to the retention values obtained for the same ions in conditions where no oil or surfactant were present (Figure 7). At the same time, $\mathrm{SO}_{4}^{2-}$ retentions lower than those for the pure solutions are observed. This change in retention behavior likely stems from interaction of the cationic surfactant with the multilayer. This may lead to an increase in $\mathrm{Ca}^{2+}$ rejection and a decrease in $\mathrm{SO}_{4}^{2-}$ retention. The increase in $\mathrm{Cl}^{-}$retention is a consequence of the electroneutrality principle: if more $\mathrm{Ca}^{2+}$ is retained, then also more of its counterion is retained.

When the anionic SDS is used as surfactant (Figure 9B), the membranes still show excellent oil retention (98\%) and good TOC retention (83\%). This time, we can observe a $\mathrm{SO}_{4}^{2-}$ retention similar to the values obtained without surfactant and previously shown in Figure 9, while we have higher $\mathrm{Ca}^{2+}$ (73\%) retention. SDS is smaller than CTAB. This possibly allows SDS easier diffusion into the multilayer than $C T A B$, as supported by a lower TOC retention for SDS. Diffusion into and adsorption of SDS to the multilayer not only brings a change in membrane surface charge, but it could also densify the layer, reducing the effective multilayer pore size and thereby also increasing divalent ion retention.

Table 1 contains a summary of the results obtained by filtrating artificial PW emulsions with our HF membranes. For CTAB stabilized PW, the membranes showed a low flux decline (just 19\%). Furthermore, after cleaning with a CTAB solution at its $\mathrm{CMC}$ for $15 \mathrm{~min}$, the membranes were able to 

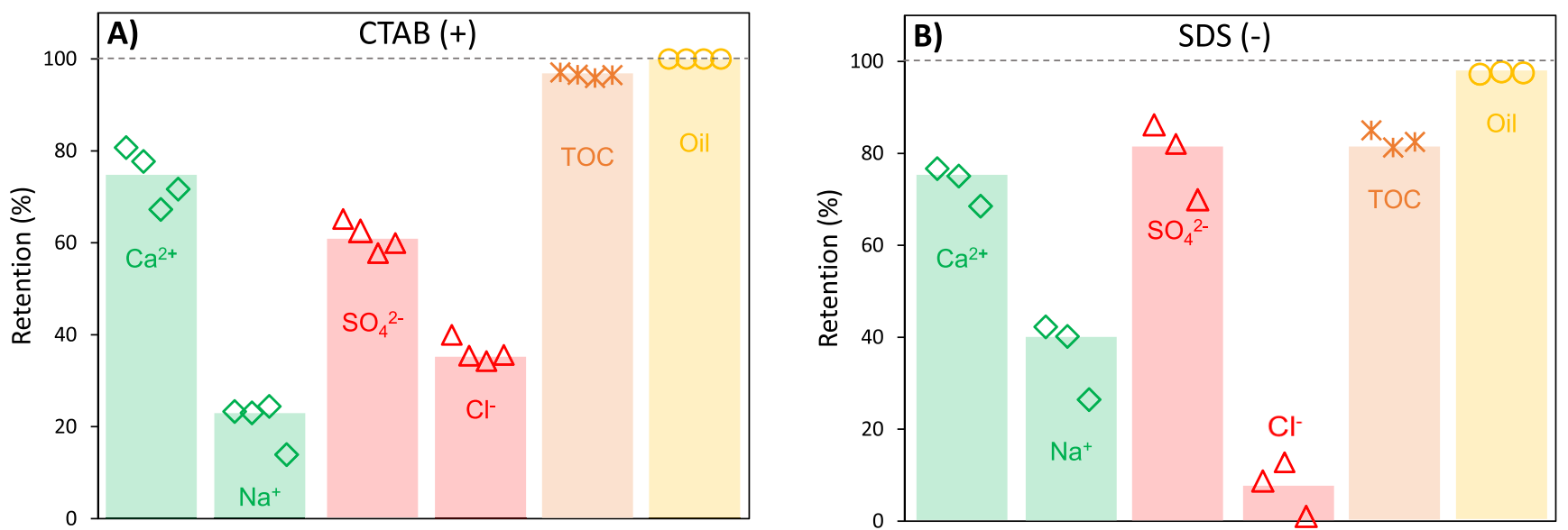

Figure 9. Retentions of $\mathrm{Ca}^{2+}, \mathrm{Na}^{+}, \mathrm{SO}_{4}^{2-}, \mathrm{Cl}^{-}$, TOC, and oil from membranes coated with (PAH/PSS) ${ }_{4}-\mathrm{PAH}$ in experiments performed with $\mathrm{O} / \mathrm{W}$ emulsions stabilized by (A) CTAB and (B) SDS. Emulsions were made of $5 \mathrm{mM} \mathrm{CaCl}, 5 \mathrm{mM} \mathrm{NaCl}, 5 \mathrm{mM} \mathrm{Na}_{2} \mathrm{SO}_{4}, 1000$ ppm of $n$-hexadecane (oil), and surfactant at its CMC. Points represent single data points, while bars represent the average of these points.

Table 1. Remaining Membrane Flux after Fouling and Flux Recovery after Cleaning of the Artificial Produced Water Experiments $^{a}$

$\begin{array}{ccc}\text { surfactant used } & \text { flux after fouling (\%) } & \text { flux recovery (\%) } \\ \text { CTAB (+) } & 81 & 100 \\ \text { SDS (-) } & 10 & 80\end{array}$

${ }^{a}$ Emulsions were made of $5 \mathrm{mM} \mathrm{CaCl}_{2}, 5 \mathrm{mM} \mathrm{NaCl}, 5 \mathrm{mM} \mathrm{Na}_{2} \mathrm{SO}_{4}$, $1000 \mathrm{mg} / \mathrm{L}$ of $n$-hexadecane (oil), and surfactant at its CMC.

completely recover their original flux. This demonstrates that fouling was not severe and that it was $100 \%$ reversible. For SDS, the situation was a bit different. Flux decline was much higher, reaching a value of $90 \%$. Still, it was possible to recover $80 \%$ of the original flux. The nonreversible part of the fouling could stem from SDS penetrating the PEM layer, as discussed above. It is also important to mention that cationic surfactants are good corrosion inhibitors and are much more commonly found in PW than anionic surfactants such as SDS. ${ }^{34}$ Overall, these results clearly show that these newly developed membranes would be highly suitable to treat PW stabilized by cationic surfactants such as CTAB and would still be suitable to treat PW stabilized by anionic surfactants. The difference in fouling behavior could stem from the easier diffusion of SDS into the multilayer, as discussed above. In both cases, the membrane cleanability was extremely good, proving that PEM deposition can lead to surfaces where fouling is less severe and easier to remove. These membranes allow deoiling and removal of most of the surfactants and divalent ions from the waste stream and even some of the monovalent ions in only one step. All of these factors together make them highly suitable for treatment of PW.

\section{CONCLUSIONS}

Membrane technology can be considered a promising technology for PW treatment. Through use of membranes, it becomes possible to convert a complex waste stream such as PW into a source of water for reuse. In particular, polyelectrolyte multilayer deposition is one of the methods that established itself as an effective technique to prepare hollow fiber-based NF membranes. Hollow fiber membranes can withstand a much higher fouling load compared to their spiral wound counterparts and would thus be especially promising for PW treatment. Unfortunately, surfactants, naturally present in PW but also added in extra quantities to inhibit pipe corrosion and increase oil recovery, can affect the stability of polyelectrolyte multilayers. In this work, we studied the stability of polyelectrolyte multilayers toward different types of surfactant (anionic, cationic, zwitterionic, and nonionic) and demonstrate that it is possible to produce stable multilayers, resistant toward surfactants, on model surfaces as well as on membranes. This is, however, only true for the cross-linkable PAH/PSS system and not for the chemically stable PDADMAC/PSS system. The effect of crosslinking time for PAH/PSS was also studied for PEM-based hollow fiber NF membranes. With increased cross-linking time, more stable membranes are produced with better separation properties but at a lower water permeability. Novel HF membranes based on 4.5 bilayers of PAH/PSS and crosslinked for $5 \mathrm{~h}$ with a $7.5 \mathrm{mM}$ glutaraldehyde solution showed excellent stability toward charged surfactants. These membranes were tested with two artificial PW emulsions stabilized either by the cationic CTAB or the anionic SDS. For CTAB stabilized PW, the membranes showed a low flux decline (just 19\%) and high surfactant removal (TOC retention 96.5\%). Furthermore, after cleaning with a CTAB solution at its CMC, the membranes were able to completely recover their original flux. For SDS flux, decline was much higher, reaching a value of $90 \%$, and surfactant removal was lower (TOC retention $83 \%$ ). Still, it was possible to recover $80 \%$ of the original flux after cleaning with SDS solution at its CMC. SDS is smaller than $\mathrm{CTAB}$, and this possibly allows SDS easier diffusion into the multilayer than $\mathrm{CTAB}$, as supported by a lower TOC retention for SDS and its higher flux decline. In both cases, the membranes also showed excellent oil removal $(\sim 100 \%)$ and divalent ion retention $\left(\sim 75 \%\right.$ for $\mathrm{Ca}^{2+}$ and up to $\sim 80 \%$ for $\mathrm{SO}_{4}^{2-}$ ). In conclusion, these membranes not only show good stability and cleanability but also allow deoiling and removal of small organic molecules (such as surfactants) and divalent ions in a single step process. 


\section{ASSOCIATED CONTENT}

\section{S Supporting Information}

The Supporting Information is available free of charge on the ACS Publications website at DOI: 10.1021/acsapm.9b00503.

SEM images of bare and coated HF membranes (PDF)

\section{AUTHOR INFORMATION}

\section{Corresponding Author}

*E-mail: w.m.devos@utwente.nl.

\section{ORCID}

Ettore Virga: 0000-0002-9304-3784

Wiebe M. de Vos: 0000-0002-0133-1931

\section{Notes}

The authors declare no competing financial interest.

\section{ACKNOWLEDGMENTS}

This work was performed in the cooperation framework of Wetsus, European Centre of Excellence for Sustainable Water Technology (www.wetsus.nl). Wetsus is cofunded by the Dutch Ministry of Economic Affairs and Ministry of Infrastructure and Environment, the European Union Regional Development Fund, the Province of Fryslân, and the Northern Netherlands Provinces. This work is part of a project that has received funding from the European Union's Horizon 2020 research and innovation programme under the Marie Skłodowska-Curie Grant 665874. The authors thank the participants of the research theme "Concentrates" for fruitful discussions and financial support.

\section{REFERENCES}

(1) Gerland, P.; Raftery, A. E.; Ševčíková, H.; Li, N.; Gu, D.; Spoorenberg, T.; Alkema, L.; Fosdick, B. K.; Chunn, J.; Lalic, N.; Bay, G.; Buettner, T.; Heilig, G. K.; Wilmoth, J. World Population Stabilization Unlikely This Century. Science 2014, 346, 234-237.

(2) Springmann, M.; Clark, M.; Mason-D’Croz, D.; Wiebe, K.; Leon Bodirsky, B.; Lassaletta, L.; Vries, W.; Vermeulen, S. J.; Herrero; Willett, W. Options for Keeping the Food System within Environmental Limits. Nature 2018, 562, 519-525.

(3) Kummu, M.; Guillaume, J.; de Moel, H.; Eisner, S.; Floerke, M.; Porkka, M.; Siebert, S.; Veldkamp, T. I.; Ward, P. The World's Road to Water Scarcity: Shortage and Stress in the 20th Century and Pathways towards Sustainability. Sci. Rep. 2016, 6, 38495.

(4) Alley, B.; Beebe, A.; Rodgers, J.; Castle, J. W. Chemical and Physical Characterization of Produced Waters from Conventional and Unconventional Fossil Fuel Resources. Chemosphere 2011, 85, 74-82.

(5) Scanlon, B.; Reedy, R.; Male, F.; Walsh, M. Water Issues Related to Transitioning from Conventional to Unconventional Oil Production in the Permian Basin. Environ. Sci. Technol. 2017, 51, 10903-10912.

(6) Igunnu, E. T.; Chen, G. Z. Produced Water Treatment Technologies. Int. J. Low-Carbon Technol. 2014, 9, 157-177.

(7) Siagian, U. W. R.; Widodo, S.; Khoiruddin, K.; Wardani, A.; Wenten, I. G. Oilfield Produced Water Reuse and Reinjection with Membrane. MATEC Web Conf. 2018, 156, 08005.

(8) Fakhru'l-Razi, A.; Pendashteh, A.; Abdullah, L. C.; Biak, D. R. A.; Madaeni, S. S.; Abidin, Z. Z. Review of Technologies for Oil and Gas Produced Water Treatment. J. Hazard. Mater. 2009, 170, 530-551.

(9) Cheryan, M.; Rajagopalan, N. Membrane Processing of Oily Streams: Wastewater Treatment and Waste Reduction. J. Membr. Sci. 1998, 151, 13-28.

(10) Dickhout, J. M.; Moreno, J.; Biesheuvel, P. M.; Boels, L.; Lammertink, R. G. H.; de Vos, W. M. Produced Water Treatment by Membranes: A Review from a Colloidal Perspective. J. Colloid Interface Sci. 2017, 487, 523-534.
(11) Bilstad, T.; Espedal, E. Membrane Separation of Produced Water. Water Sci. Technol. 1996, 34, 239.

(12) Kang, G.-d.; Cao, Y.-m Development of Antifouling Reverse Osmosis Membranes for Water Treatment: A Review. Water Res. 2012, 46, 584-600.

(13) Naidu, D. L.; Saravanan, S.; Chidambaram, M.; Goel, M.; Das, A.; Sarat Chandra Babu, J. Nanofiltration in Transforming Surface Water into Healthy Water: Comparison with Reverse Osmosis. J. Chem. 2015, 2015, 1-6.

(14) Alzahrani, S.; Mohammad, A. Challenges and Trends in Membrane Technology Implementation for Produced Water Treatment: A Review. J. Water Process Eng. 2014, 4, 107-133.

(15) Vrouwenvelder, J.; von der Schulenburg, D. G.; Kruithof, J.; Johns, M.; van Loosdrecht, M. Biofouling of Spiral-Wound Nanofiltration and Reverse Osmosis Membranes: A Feed Spacer Problem. Water Res. 2009, 43, 583-594.

(16) de Grooth, J.; Haakmeester, B.; Wever, C.; Potreck, J.; de Vos, W.; Nijmeijer, K. Long Term Physical and Chemical Stability of Polyelectrolyte Multilayer Membranes. J. Membr. Sci. 2015, 489, 153159.

(17) de Grooth, J.; Oborny, R.; Potreck, J.; Nijmeijer, K.; de Vos, W. The Role of Ionic Strength and Odd-Even Effects on the Properties of Polyelectrolyte Multilayer Nanofiltration Membranes. J. Membr. Sci. 2015, 475, 311-319.

(18) Ilyas, S.; Abtahi, S. M.; Akkilic, N.; Roesink, H.; de Vos, W. Weak Polyelectrolyte Multilayers as Tunable Separation Layers for Micro-Pollutant Removal by Hollow Fiber Nanofiltration Membranes. J. Membr. Sci. 2017, 537, 220-228.

(19) Ji, Y.-L.; Gu, B.-X.; An, Q.-F.; Gao, C.-J. Recent Advances in the Fabrication of Membranes Containing "Ion Pairs" for Nanofiltration Processes. Polymers 2017, 9, 715.

(20) Al-Zoubi, H.; Omar, W. Rejection of Salt Mixtures from High Saline by Nanofiltration Membranes. Korean J. Chem. Eng. 2009, 26, 799-805.

(21) Kwon, Y.-N.; Leckie, J. O. Hypochlorite Degradation of Crosslinked Polyamide Membranes: II. Changes in Hydrogen Bonding Behavior and Performance. J. Membr. Sci. 2006, 282, 456464.

(22) Kang, J.; Dahne, L. Strong Response of Multilayer Polyelectrolyte Films to Cationic Surfactants. Langmuir 2011, 27, 4627-4634.

(23) An, Q.; Huang, T.; Shi, F. Covalent Layer-by-Layer Films: Chemistry, Design, and Multidisciplinary Applications. Chem. Soc. Rev. 2018, 47, 5061-5098.

(24) Duong, P. H.; Zuo, J.; Chung, T.-S. Highly Crosslinked Layerby-Layer Polyelectrolyte FO Membranes: Understanding Effects of Salt Concentration and Deposition Time on FO Performance. J. Membr. Sci. 2013, 427, 411-421.

(25) Cho, K. L.; Hill, A. J.; Caruso, F.; Kentish, S. E. Chlorine Resistant Glutaraldehyde Crosslinked Polyelectrolyte Multilayer Membranes for Desalination. Adv. Mater. 2015, 27, 2811.

(26) Dijt, J. C.; Stuart, M. A. C.; Fleer, G. J. Reflectometry as a Tool for Adsorption Studies. Adv. Colloid Interface Sci. 1994, 50, 79-101.

(27) Dickhout, J.; Kleijn, M.; Lammertink, R. G. H.; de Vos, W. Adhesion of Emulsified Oil Droplets to Hydrophilic and Hydrophobic Surfaces - Effect of Surfactant Charge, Surfactant Concentration and Ionic Strength. Soft Matter 2018, 14, 5452-5460.

(28) Bazyar, H.; Lv, P.; Wood, J.; Porada, S.; Lohse, D.; Lammertink, R. Liquid-Liquid Displacement in Slippery LiquidInfused Membranes (SLIMs). Soft Matter 2018, 14, 1780-1788.

(29) Antonietti, M.; Conrad, J.; Thuenemann, A. PolyelectrolyteSurfactant Complexes: ANew Type of Solid, Mesomorphous Material. Macromolecules 1994, 27, 6007-6011.

(30) Israelachvili, J. N. In Intermolecular and Surface Forces, 3rd ed.; Israelachvili, J. N., Ed.; Academic Press: Boston, 2011; pp 151-167.

(31) Takigawa, T.; Endo, Y. Effects of Glutaraldehyde Exposure on Human Health. J. Occup. Health 2006, 48, 75-87.

(32) Park, J.; Park, J.; Kim, S. H.; Cho, J.; Bang, J. Desalination Membranes from $\mathrm{pH}$-Controlled and Thermally-Crosslinked Layer- 
by-Layer assembled Multilayers. J. Mater. Chem. 2010, 20, 20852091.

(33) Virga, E.; de Vos, W. M.; Biesheuvel, P. M. Theory of Gel Expansion to Generate Electrical Energy. EPL 2017, 120, 46002.

(34) Migahed, M.; Al-Sabagh, A. Beneficial Role of Surfactants as Corrosion Inhibitors in Petroleum Industry: A Review Article. Chem.

Eng. Commun. 2009, 196, 1054-1075. 